\title{
PET scanning and neuronal loss in acute vegetative state
}

\author{
*S Laureys, ME Faymonville, G Moonen, A Luxen, P Maquet \\ *Cyclotron Research Centre, University of Liège, B-4000 Liège 1, Belgium; Department of Neurology, and \\ Anaesthesiology and Intensive Care Medicine, CHU Sart Tilman, Liège, Belgium
}

Sir J Rudolf and colleagues (Jan 8, p 115) ${ }^{1}$ used ${ }^{11}$ C-flumazenil positron emission tomography (PET) to identify irreversible structural brain damage in nine patients with postanoxic vegetative state. Only one of these patients subsequently recovered responsiveness although remaining almost totally dependent. The investigators conclude that the quantification of the extent of neuronal damage using ${ }^{11} \mathrm{C}$-flumazenil PET predicted the possibility of recovery of consciousness. We congratulate the authors for this first description of a potentially useful tool in evaluating residual brain function in patients in a vegetative state. However, we would like to make two comments.

First, the degree of surviving neurones is not the only critical parameter on which the outcome of vegetative patients depends. Indeed, unpublished results of a PET study currently in progress in our centre show that in patients who recover consciousness after being in a postanoxic vegetative state, glucose metabolism is partially restored in discrete cortical regions. ${ }^{2}$ So far, out of 14 patients studied, three patients recovered consciousness and could be rescanned. In these three patients, we localised the brain areas where the metabolism was most impaired during the vegetative state and returned to near-normal values after recovery. The control population consisted of 35 age-matched healthy individuals. This analysis identified bilateral prefrontal cortices (Brodmann's area 8, 9 and 10), Broca's area (left area 44/45), left superior temporal gyrus (area 22), bilateral inferior parietal lobule (area 39 and 40) and precuneus (area 7 and 31). We hypothesise that the resumption of long-range functional connectivity between these associative cortices and between some of these and the thalami plays an important role in the restoration of their functional integrity. ${ }^{3}$ The cellular mechanisms which underlie this functional normalisation remain putative: axonal sprouting, neurite outgrowth, cell division (known to occur predominantly in associative cortices in normal primates $)^{4}$ or even apoptosis. This residual cerebral plasticity during vegetative state has been largely overlooked and deserves further investigation. We believe that the challenge is now to identify the conditions in which and the mechanisms by which some patients may recover consciousness rather than to consider only the amount of neuronal loss. In this respect, we would like to ask the investigators whether they had the opportunity to rescan their patient who recovered.

Second, great caution is required before proposing to the medical community any procedure with a prognostic value in vegetative state. In our view, the interesting data reported by Rudolf and colleagues do not allow to claim that ${ }^{11} \mathrm{C}$-flumazenil PET has such prognostic value because, unfortunately, no data are given about the differences between the patient who subsequently recovered and the eight other patients who died or remained in a vegetative state.

\section{References}

1. Rudolf J, Sobesky J, Grond M, Heiss WD. Identification by positron emission tomography of neuronal loss in acute vegetative state. Lancet 2000; 355: 115-16.

2. Laureys S, Lemaire C, Maquet P, Phillips C, Franck G. Cerebral metabolism during vegetative state and after recovery to consciousness. J Neurol Neurosurg Psychiatry 1999; 67: 121.

3. Laureys S, Faymonville ME, Luxen A, Lamy M, Franck G, Maquet P. Restoration of thalamocortical connectivity after recovery from persistent vegetative state. Lancet (in press).

4. Gould E, Reeves AJ, Graziano MS, Gross CG. Neurogenesis in the neocortex of adult primates. Science 1999; 286(5439): 548-52. 